ISSN 1991-8631

Original Paper

http://indexmedicus.afro.who.int

\title{
Évaluation de la pollution physico-chimique des eaux de l'Oued Khoumane (Moulay Idriss Zerhoun, Maroc)
}

\author{
Ali BEN MOUSSA *, Abdelkader CHAHLAOUI et Habib ROUR El \\ Équipe de Gestion et Valorisation des Ressources Naturelles, Laboratoire de l'Environnement et Santé, \\ Faculté des Sciences de Meknès, Département de Biologie. Université Moulay Ismail, BP 11201 Zitoune \\ Meknès, Maroc. \\ *Auteur correspondant,E-mail: alidoctorant@gmail.com ; alidosya@yahoo.fr; Tel :(212) 0611933736.
}

\section{RESUMÉ}

L'Oued Khoumane est un cours d'eau traversant du sud-est à l'ouest la ville de Moulay Idriss Zerhoun. L'intérêt porté à ce cours d'eau réside essentiellement dans le fait qu'il reçoit les eaux usées non traitées de la ville et que ses eaux sont utilisées en irrigation des cultures. Le présent travail a pour objectif l'étude de la qualité physico-chimique des eaux de l'Oued au cours de la période d'août 2010 à juillet 2011. Les résultats obtenus ont montré que la qualité de ces eaux présente un degré de pollution organique inquiétant et une minéralisation importante et que la source thermale Ain Hamma Moulay Idriss contribue considérablement dans leur minéralisation. L'Analyse explicative, par Analyse en Composantes Principales (ACP) des données physico-chimiques, nous a permis de déceler des corrélations entre les différents paramètres et de différencier une typologie de la qualité de l'eau dans la région d'étude. Les résultats font ressortir une situation alarmante de la qualité des eaux de l'Oued. Afin, de résoudre ce problème, nous recommandons un aménagement de la source thermale Ain Hamma Moulay Idriss, un réseau d'assainissement adéquat des eaux usées brutes et une gestion convenable des rejets émis par les huileries.

(C) 2012 International Formulae Group. All rights reserved.

Mots clés: Minéralisation, qualité, irrigation, volubilis, Meknès.

\section{INTRODUCTION}

La détérioration de la qualité des ressources en eau constitue une menace aussi importante que celle liée au déséquilibre quantitatif (El addouli et al., 2009a). Les sources de pollution des eaux sont diverses et multiples. Les eaux souterraines, souvent protégées géologiquement, sont exposées à des différentes sortes de pollution (Bricha, 2007). Les eaux superficielles elles aussi, sont menacées par les rejets des polluants: industriels, domestiques, agricoles, atmosphériques, thermiques. Les activités anthropiques sont à l'origine de la plupart des problèmes de pollution des cours d'eau.

Au Maroc, les écoulements superficiels sont tributaires des précipitations et présentent une grande variabilité spatiale (Bouaicha et Benabdelfadel, 2010). En général, les ressources hydriques dont dispose le Maroc sont limitées et soumises à des variations cycliques extrêmes (Alibou, 2002). De même, la situation qualitative des eaux est loin d'être satisfaisante (Chahlaoui et al., 1997).

A l'échelle de la province de Meknès, située au centre du Maroc, le réseau 
hydrographique superficiel est constitué essentiellement par les Oueds: Boufekrane, Ouislane, Bouishak et Khoumane. A l'exception de ce dernier, les trois premiers cours d'eau ont suscité l'intérêt de nombreux scientifiques (Chahlaoui, 1996; Karrouch et Chahlaoui, 2007; Aboulkacem et al., 2007; Karrouch et Chahlaoui, 2009; El addouli et al., 2009b; El addouli, 2010; Lamrani et al., 2011; El addouli et al., 2012; Lamrani et al., 2012).

A $25 \mathrm{~km}$ de Meknès, se situe la ville touristique de Moulay Idriss Zerhoun. Elle est implantée sur le relief rocheux qui domine la vallée de l'Oued Khoumane. A l'instar de plusieurs villes marocaines, cette ville est confrontée à des difficultés d'assainissement de ses eaux usées brutes. Ces difficultés sont liées notamment à l'évacuation, sans aucun traitement préalable, de ces eaux dans l'Oued Khoumane. L'intérêt porté sur ce cours d'eau réside essentiellement dans le fait qu'il reçoit les effluents domestiques de la ville, les eaux thermales de la source Ain Hamma Moulay Idriss et les margines pendant la période de la trituration d'olive. De plus, ses eaux sont utilisées en irrigation, en baignade, en abreuvement du cheptel et dans des activités domestiques de certaines populations environnantes.

Cet état du lieu, peut générer un déséquilibre sérieux de cet écosystème aquatique et être à

l'origine des nuisances pour la population humaine et pour la faune et la flore. Un diagnostic de la situation actuelle de la pollution et un suivi spatio-temporel rigoureux de son évolution, s'avèrent d'une grande importance pour la sauvegarde de cet écosystème.

Ce travail réalisé sur l'Oued Khoumane est original, dans la mesure où aucune recherche hydrobiologique n'a été réalisée auparavant et que cet Oued, par le biais de l'ensemble des polluants qu'il charrie, pourrait constituer un danger pour la santé et l'environnement. Il s'intéresse à l'étude de la qualité de l'Oued, en réalisant un suivi spatio- temporel des paramètres physico-chimiques des eaux échantillonnées, afin de préciser l'importance de cette pollution.

\section{MATERIEL ET METHODES}

Zone d'étude et stations d'échantillonnage

Moulay Idriss Zerhoun est une ville touristique et spirituelle située à $25 \mathrm{Km}$ de Meknès. Elle est traversée du sud-est à l'ouest par l'Oued Khoumane. Ce dernier est un cours d'eau alimenté par des sources d'eau, dont la plus caractéristique est la source thermale Ain Hamma Moulay Idriss. De plus, il constitue le seul milieu récepteur des effluents des eaux usées domestiques de la ville.

Au total, neuf stations ont été choisies : Sept stations (S1, S2, S3, S4, S5, S6 et S7) situées sur le lit de 1'Oued et deux (A1 et A2) correspondent à des stations ponctuelles au niveau de deux sources importantes. Le choix des stations sur l'Oued a tenu compte du sens d'écoulement amont-aval de l'eau, du déversement des eaux de sources et de la proximité des rejets polluants. Ces points de prélèvement sont de l'amont vers l'aval (Figure 1):

A1 : Borne Fontaine Ain Chench alimentant considérablement l'Oued en amont;

S1 : située plus en amont et juste en aval immédiat du ruisseau provenant de la station A1;

S2 : située en amont immédiat de la confluence de l'Oued avec les eaux thermales de la source Ain Hamma Moulay Idriss;

A2: correspond à la source thermale Ain Hamma Moulay Idriss;

S3: se trouve en aval immédiat de la confluence de l'Oued avec les eaux thermales; S4: se situe en aval d'un premier exutoire important des eaux résiduaires et d'une décharge non contrôlée;

S5: se localise en aval d'un deuxième exutoire et des rejets du fumier et du lisier ;

S6 : se situe juste en aval du dernier exutoire important (aval de la ville);

S7 : se situe plus en aval de l'Oued et de la ville historique volubilis. 


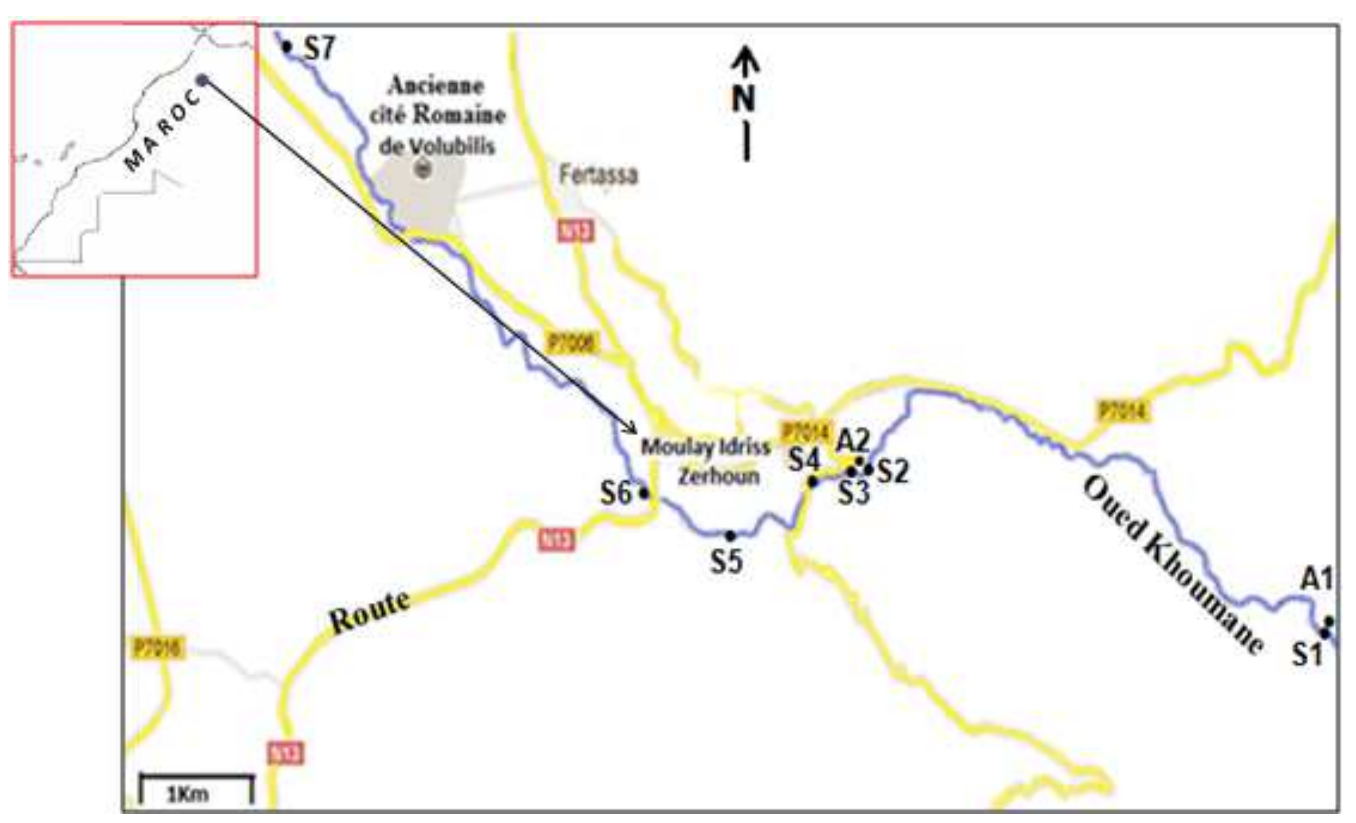

Figure 1: Situation des stations d'échantillonnage.

Prélèvements et analyses physico-chimiques L'échantillonnage de l'eau a été effectué mensuellement au cours d'un cycle d'étude compris entre août 2010 et juillet 2011. L'étude a porté sur la mesure in situ du $\mathrm{pH}$ (par un pH-mètre), de la conductivité et la salinité (par un conductimètre portable de type Consort K912), du débit, de la température de l'air et de l'eau (par un thermomètre) et sur la détermination au laboratoire d'autres éléments majeurs : Oxygène dissous, Sulfates, Chlorures, Dureté totale, Nitrates, Nitrites, Orthophosphates, Demande Chimique en Oxygène, Demande Biologique en Oxygène et les matières en suspension. Le transport des échantillons s'est effectué dans une glaciaire à $4{ }^{\circ} \mathrm{C}$, et les analyses ont été réalisées selon des méthodes homologuées par Rodier (2009).

\section{Analyse des données}

Les données physico-chimiques obtenues ont été traitées par Analyses en Composantes Principales (ACP) en utilisant le logiciel STATISTICA version 2009, avec un seuil de signification (risque d'erreur) de 5\%.

\section{RÉSULTATS}

Analyse descriptive

Les variations spatio-temporelles des paramètres physico-chimiques étudiés sont illustrées sur les Figures 2 et 3 .

Les températures de l'air les plus élevées ont été enregistrées en été $\left(22,9^{\circ} \mathrm{C}\right.$ à $27,9^{\circ} \mathrm{C}$ ). Alors que les plus basses en hiver $\left(11,7{ }^{\circ} \mathrm{C}\right.$ à $\left.15,8{ }^{\circ} \mathrm{C}\right)$ et les températures intermédiaires en automne $\left(19,3{ }^{\circ} \mathrm{C}\right.$ à $\left.20,9^{\circ} \mathrm{C}\right)$ et en printemps $\left(18,2^{\circ} \mathrm{C}\right.$ à $\left.23,6^{\circ} \mathrm{C}\right)$.

La température moyenne de l'eau des sources A1 et A2 marque respectivement $17,66 \pm 2,15{ }^{\circ} \mathrm{C}$ et $30,9 \pm 1,57{ }^{\circ} \mathrm{C}$. Celle de l'Oued, marque $18,8 \pm 5,22{ }^{\circ} \mathrm{C}$ en $\mathrm{S} 1$ et augmente le long de l'Oued pour atteindre un pic maximal $\left(24,84 \pm 6,42{ }^{\circ} \mathrm{C}\right)$ en $\mathrm{S} 3$ puis diminue vers l'aval $\left(20,47 \pm 4,40{ }^{\circ} \mathrm{C}\right.$ en S7). Les variations temporelles de ce paramètre au niveau des eaux de l'Oued sont remarquables : les plus basses $\left(12{ }^{\circ} \mathrm{C}\right.$ à $\left.15,87{ }^{\circ} \mathrm{C}\right)$ sont enregistrées en hiver, les plus élevées en été $\left(22,57^{\circ} \mathrm{C}\right.$ à $\left.30,23{ }^{\circ} \mathrm{C}\right)$ et en automne $\left(20,33{ }^{\circ} \mathrm{C}\right.$ à $29,17^{\circ} \mathrm{C}$ ); et les valeurs intermédiaires $\left(17,13^{\circ} \mathrm{C}\right.$ à $\left.24,3^{\circ} \mathrm{C}\right)$ sont notées en printemps. 
Les températures mensuelles sont comprises entre $11{ }^{\circ} \mathrm{C}$ enregistrée en $\mathrm{S} 1$ et $30,5^{\circ} \mathrm{C}$ en S3.

Les valeurs moyennes du $\mathrm{pH}$ des eaux de l'Oued se trouvent dans la zone légèrement acide à neutre, variant de $6,5 \pm 0,47$ à $6,75 \pm 0,42$, et dévoilent un léger gradient décroissant de $\mathrm{S} 1$ à $\mathrm{S} 3$ puis un autre légèrement croissant vers l'aval. Les valeurs enregistrées dans les deux sources A1 et A2 sont de l'ordre de $6,73 \pm 0,49$ et $6,31 \pm 0,57$ respectivement. Les valeurs saisonnières des eaux analysées ne présentent pas de variations très importantes.

La variation spatio-temporelle du débit moyen annuel de l'Oued Khoumane met en général en évidence un gradient croissant de l'amont $(104,23$ 1/s en S1) vers l'aval $(365,64$ 1/s en S7). Le débit le plus élevé a été enregistré en hiver, atteignant une moyenne saisonnière d'ordre de 602,73 1/s et d'environ $256,41 / \mathrm{s}$ en printemps. Par contre, en été et en automne, il est plus bas en marquant respectivement 90,70 1/s et 88,96 1/s (comme moyenne saisonnière de toutes les stations de l'Oued). Selon l'importance du débit, Nous distinguons donc trois périodes: une première période estivo- automnale avec des débits faibles, une seconde hivernale avec des débits élevés et une troisième correspondant au printemps avec un débit moyen. Pour les sources étudiées, le débit moyen de la source thermale $(9,83 \mathrm{l} / \mathrm{s})$ est plus important que celui de la borne fontaine A1 (0,77 1/s).

La conductivité dans la station A1 est de l'ordre de $572,42 \pm 28,78 \mu \mathrm{s} / \mathrm{cm}$. Les eaux de l'Oued sont caractérisées par une conductivité moyenne très élevée. Elle augmente de S1 $(652,75 \pm 194,67 \mu \mathrm{s} / \mathrm{cm})$ vers S3 (pic maximal : $3024,83 \pm 1487,96 \mu \mathrm{s} / \mathrm{cm}$ ) et diminue de S3 vers l'aval (1623,92 $\pm 486,68$ $\mu \mathrm{s} / \mathrm{cm}$ en S7). L'Oued Khoumane passe ainsi d'une conductivité moyenne de 2024,25 $\mu \mathrm{S} / \mathrm{cm}$ en $\mathrm{S} 2$ à $3024,83 \mu \mathrm{S} / \mathrm{cm}$ en $\mathrm{S} 3$; suite à la confluence avec les eaux de la source A2 (4153,33 $\pm 300,07 \mu \mathrm{s} / \mathrm{cm})$.

L'évolution saisonnière de ce paramètre a révélé, une chute de la conductivité des eaux de l'Oued en hiver (583 à $1166 \mu \mathrm{s} / \mathrm{cm})$ et en printemps $(537,67$ à 2180 $\mu \mathrm{s} / \mathrm{cm})$ par rapport aux valeurs mesurées en été $(611,33 \mu \mathrm{s} / \mathrm{cm}$ à $4276,67 \mu \mathrm{s} / \mathrm{cm})$ et en automne $(879 \mu \mathrm{s} / \mathrm{cm}$ à 4476,67 $\mu \mathrm{s} / \mathrm{cm})$.

La salinité moyenne des eaux de l'Oued Khoumane oscille entre $122 \mathrm{mg} / \mathrm{l}$ et $2440 \mathrm{mg} / \mathrm{l}$ comme valeurs extrêmes minimales et maximales enregistrées respectivement dans S1 et S3. L'évolution spatiale et saisonnière de la salinité des eaux analysées rappelle celle de la conductivité.

L'évolution spatiale de la dureté totale moyenne a révélé un gradient croissant de $\mathrm{S} 1\left(29,87 \pm 10,45^{\circ} \mathrm{F}\right)$ vers S3 $(125,03 \pm 65,67$ $\left.{ }^{\circ} \mathrm{F}\right)$ puis décroissant de $\mathrm{S} 3$ vers $\mathrm{S} 7$ $\left(56,05 \pm 15,31^{\circ} \mathrm{F}\right)$. Les teneurs les plus élevées ont été enregistrées en été $\left(29,33^{\circ} \mathrm{F}\right.$ à 174,80 $\left.{ }^{\circ} \mathrm{F}\right)$ et en automne $\left(39^{\circ} \mathrm{F}\right.$ à $\left.194,67^{\circ} \mathrm{F}\right)$, alors que les valeurs de moins de $46,5^{\circ} \mathrm{F}$ et de $84,13^{\circ} \mathrm{F}$ sont enregistrées respectivement en hiver et en printemps. Au niveau des sources A1 et A2, les valeurs moyennes enregistrées sont de l'ordre de $22,41 \pm 5,07{ }^{\circ} \mathrm{F}$ et $190,58 \pm 21,04{ }^{\circ} \mathrm{F}$ respectivement.

Les teneurs moyennes en chlorures augmentent de l'amont vers l'aval en montrant des variations importantes entre les stations: des teneurs minimales en amont (73,47 $\left.\pm 27,42 \mathrm{mg} \mathrm{cl}^{-} / 1\right)$, un pic maximal en S3 $(395,83 \pm 214,55 \mathrm{mg} \mathrm{cl} / 1)$ et des teneurs intermédiaires plus en aval $(198,80 \pm 83,05 \mathrm{mg}$ $\mathrm{cl}^{-} / 1$ en S7). Les teneurs moyennes enregistrées dans A1 et A2 sont respectivement de l'ordre de $52,94 \pm 16 \mathrm{mg} \mathrm{cl}^{-}$ $/$ et $547,97 \pm 75,56 \mathrm{mg} \mathrm{cl}^{-} / 1$. L'évolution spatio-temporelle des chlorures dans les eaux de l'Oued présente la même allure que celle de la salinité.

Les concentrations moyennes en sulfates dans les eaux de l'Oued augmentent de S1 $(105 \pm 74,19 \quad \mathrm{mg} / \mathrm{l})$ vers S3 $(890,44 \pm 335,68 \mathrm{mg} / \mathrm{l})$ puis diminuent de S3 vers S7 $(371,50 \pm 142,64 \quad \mathrm{mg} / \mathrm{l})$. Les fluctuations saisonnières sont bien marquées avec des concentrations élevées en été $(178,11$ à $1150,87 \mathrm{mg} / \mathrm{l})$ et en automne (80,62 à $1129,05 \mathrm{mg} / \mathrm{l})$ et une chute des teneurs en hiver $(69,93$ à $695,53 \mathrm{mg} / \mathrm{l})$ et au printemps 
(91,32 à 586,28 $\mathrm{mg} / \mathrm{l})$. La source A2 $(1099,23 \pm 211,96 \mathrm{mg} / \mathrm{l})$ est plus chargée que la borne fontaine $(145,06 \pm 73,81 \mathrm{mg} / \mathrm{l})$.

Le suivi des teneurs moyennes en nitrates montre que la source A1 $(5,47$ $\pm 2,37 \mathrm{mg} / \mathrm{l}$ ) est plus riche en nitrates que la source A2 (1,46 $\pm 0,41 \mathrm{mg} / \mathrm{l})$. Le long de l'Oued, les teneurs en nitrates évoluent selon un gradient croissant de l'amont (2,14 $\pm 0,61 \mathrm{mg} / \mathrm{l}$ en $\mathrm{S} 1)$ vers l'aval $(8,98 \pm 2,52 \mathrm{mg} / \mathrm{l}$ en S7). Selon les saisons, nous avons enregistré une augmentation des teneurs en hiver $(2,86$ à $12,49 \mathrm{mg} / \mathrm{l})$ par rapport à l'été $(1,74$ à $7,38 \mathrm{mg} / \mathrm{l})$ à l'automne $(1,52$ à 7,43 $\mathrm{mg} / \mathrm{l})$ et au printemps $(2,45$ à $8,74 \mathrm{mg} / \mathrm{l})$.

L'évolution spatiale des teneurs en nitrites suit un gradient croissant de l'amont $(1,33 \pm 0,43 \mathrm{mg} / \mathrm{l}$ en $\mathrm{S} 1)$ vers l'aval de l'Oued $(2,82 \pm 0,80 \mathrm{mg} / \mathrm{l}$ en $\mathrm{S} 7)$. La saison d'hiver a enregistré des teneurs plus importantes que les autres saisons.

Les concentrations moyennes des Orthophosphates dans les deux sources A1 et A2 sont de l'ordre de $0,79 \pm 0,57 \mathrm{mg} / \mathrm{l}$ et $0,81 \pm 0,40 \mathrm{mg} / \mathrm{l}$ respectivement. Dans les stations S1, S2 et $\mathrm{S} 3$, les teneurs en orthophosphates sont les plus faibles $(<1,75$ $\pm 0,84 \mathrm{mg} / \mathrm{l}$ ). Ces teneurs deviennent de plus en plus importantes vers l'aval en marquant 4,34 $\pm 2,35 \mathrm{mg} / \mathrm{l}$ en S6. L'évolution saisonnière de ces concentrations montre que l'hiver enregistre les valeurs les plus élevées $(0,72$ à 7,75 mg/l).

La Demande Chimique en Oxygène (DCO) évolue selon un gradient croissant de l'amont vers l'aval de l'Oued. Les valeurs moyennes sont comprises entre la valeur $70,95 \pm 19,59 \mathrm{mg} / \mathrm{l}$ enregistrée en $\mathrm{S} 1$ et $527,61 \pm 196,58 \mathrm{mg} / 1$ notée en S6. L'évolution saisonnière de la DCO montre des teneurs plus élevées en hiver $(75,47$ en $\mathrm{S} 1$ à 708,13 $\mathrm{mg} / \mathrm{l}$ en $\mathrm{S} 7)$ suivi d'automne (74,17 en $\mathrm{S} 1$ à $577,30 \mathrm{mg} / \mathrm{l}$ en $\mathrm{S} 6)$ et d'été (88,50 en S1 à 552 $\mathrm{mg} / \mathrm{l}$ en S6). En printemps, les valeurs enregistrées sont comprises entre $45,67 \mathrm{mg} / \mathrm{l}$ en $\mathrm{S} 1$ et $303,33 \mathrm{mg} / \mathrm{l}$ en $\mathrm{S} 6$.

La comparaison des valeurs moyennes révèle que la Demande Biologique en
Oxygène (DBO) suit un gradient croissant de l'amont $(6,92 \pm 3,35 \mathrm{mg} / \mathrm{l}$ en $\mathrm{S} 1)$ vers l'aval de l'Oued $(198,92 \pm 72,31 \mathrm{mg} / 1$ en $\mathrm{S} 7)$. L'évolution saisonnière de la DBO démontre qu'elle s'intensifie spectaculairement en hiver (264,83 mg/l en S7), en été (219,16 mg/l en S6) et en automne (195 mg/l en S6) et qu'elle diminue en printemps $(115,33 \mathrm{mg} / \mathrm{l}$ en $\mathrm{S} 6)$.

Les teneurs mensuelles en oxygène dissous montrent qu'elles sont faibles en été et en automne (entre 5,58 et 4,9 mg/l dans $\mathrm{S} 1$ et moins de $4 \mathrm{mg} / \mathrm{l}$ dans les autres stations) et qu'elle augmente (sans dépasser 6,8 mg/l) en printemps et en hiver. La comparaison des teneurs moyennes révèle un gradient décroissant de l'oxygène dissous de l'amont $(5,92 \pm 0,77 \mathrm{mg} / \mathrm{l}$ en $\mathrm{S} 1)$ vers l'aval $(3,29 \pm 0,9$ $\mathrm{mg} / \mathrm{l}$ en $\mathrm{S} 6)$. La source A1 est plus oxygénée $(7,06 \pm 0,40 \mathrm{mg} / \mathrm{l})$ que la source A2 $(2,95$ $\pm 0,80 \mathrm{mg} / \mathrm{l})$.

Les teneurs moyennes en matières en suspension (MES) augmentent selon un gradient croissant de l'amont $(65,77 \mathrm{mg} / \mathrm{l}$ en S1) vers l'aval $(420,39 \mathrm{mg} / \mathrm{l}$ en $\mathrm{S} 6)$. Les moyennes saisonnières enregistrées sont de l'ordre 471,27 mg/l en hiver; 249,32 mg/l en automne; $199,13 \mathrm{mg} / \mathrm{l}$ en été et 147,02 mg/l au printemps.

\section{Analyse explicative}

Cette analyse a été réalisée sur un tableau de données de neuf individus (stations) et de seize variables: Température de l'air (Tair), Température de l'eau (Teau), $\mathrm{pH}$, Salinité (Sal), Conductivité (Cond), Chlorures (cl), sulfates (SO4), Nitrates (NO3), Nitrites (NO2), Orthophosphates (PO4), Dureté totale (DT), Demande chimique en oxygène (DCO), Demande biologique en oxygène (DBO), Oxygène dissous $\left(\mathrm{O}_{2}\right)$, les Matières En Suspension (MES) et le débit (Q).

Le plan factoriel F1-F2 nous a permis d'expliquer $96,16 \%$ de la variabilité totale. L'examen des données relatives à la matrice des corrélations entre les variables nous a permis de révéler l'existence d'une forte corrélation significativement élevée entre la 
conductivité, les sulfates, les chlorures, la dureté totale, la salinité et la température de l'eau d'une part, et une corrélation significativement élevée entre la DBO et les autres parametres MES, Q, NO2, NO3 et les PO4 d'autre part.

La projection des variables sur le plan factoriel F1- F2 (Figure 4 (A)) montre que : Les températures de l'air et de l'eau, la salinité, la conductivité, les sulfates, les chlorures, et la dureté totale sont corrélées négativement avec $\mathrm{F} 1$, contrairement à l'oxygène dissous associé positivement à cet axe. Ce dernier, renseigne sur la majorité des paramètres qui déterminent le degré de la pollution minérale des eaux, et oppose donc les eaux minéralisées aux eaux faiblement ou moins chargées. Il définit alors un gradient de minéralisation allant de la droite vers la gauche de l'axe.

Les paramètres : DBO, MES, Q, NO3, $\mathrm{PO} 4$ et les NO2 sont corrélées négativement avec F2. Ce pôle regroupe donc, la majorité des paramètres qui déterminent le degré de pollution organique. Cet axe définit alors un gradient de pollution organique allant du pôle positif vers l'autre négatif de l'axe.

L'analyse de la projection des individus sur le plan factoriel F1-F2 nous a permis de définir une typologie dominée par l'individualisation de quatre groupes de stations (Figure 4 (B)):

Groupe 1 : constitué exclusivement par les stations A1 et S1 (situés plus en amont de l'Oued) corrélées positivement avec la composante F1. Les eaux de ce groupe sont plus oxygénées.

Groupe 2: c'est un groupe intermédiaire constitué par les eaux de la station S2 (moyennement oxygéné et non menacées par les rejets directs de la source A2 et des eaux domestiques de la ville Moulay Idriss Zerhoun).

Groupe 3 : constitué par la station A2 (eaux plus minéralisées et caractérisées par des températures élevées) et par l'individu S3 (eaux de l'Oued les plus minéralisées et plus influencées directement par les eaux thermales de source Ain Hamma Moulay Idriss).

Groupe 4: ce dernier groupe caractérise les individus en aval (S4, S5, S6 et S7) et correspond aux eaux de l'Oued les plus chargées en matière organique et plus menacées par les rejets domestiques de la ville.

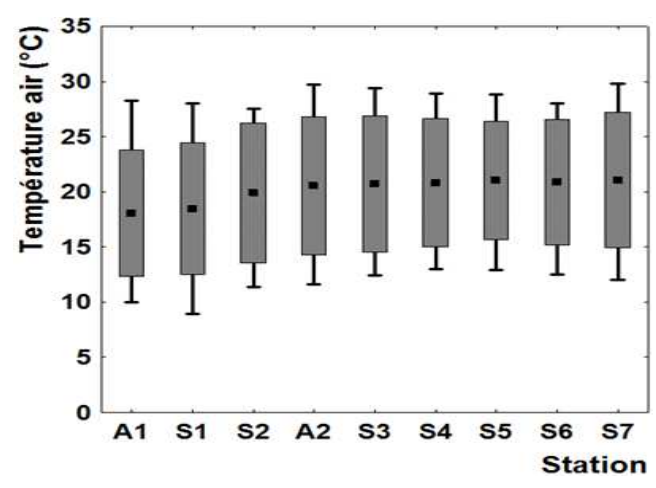


A. BEN MOUSSA et al. / Int. J. Biol. Chem. Sci. 6(6): 7096-7111, 2012
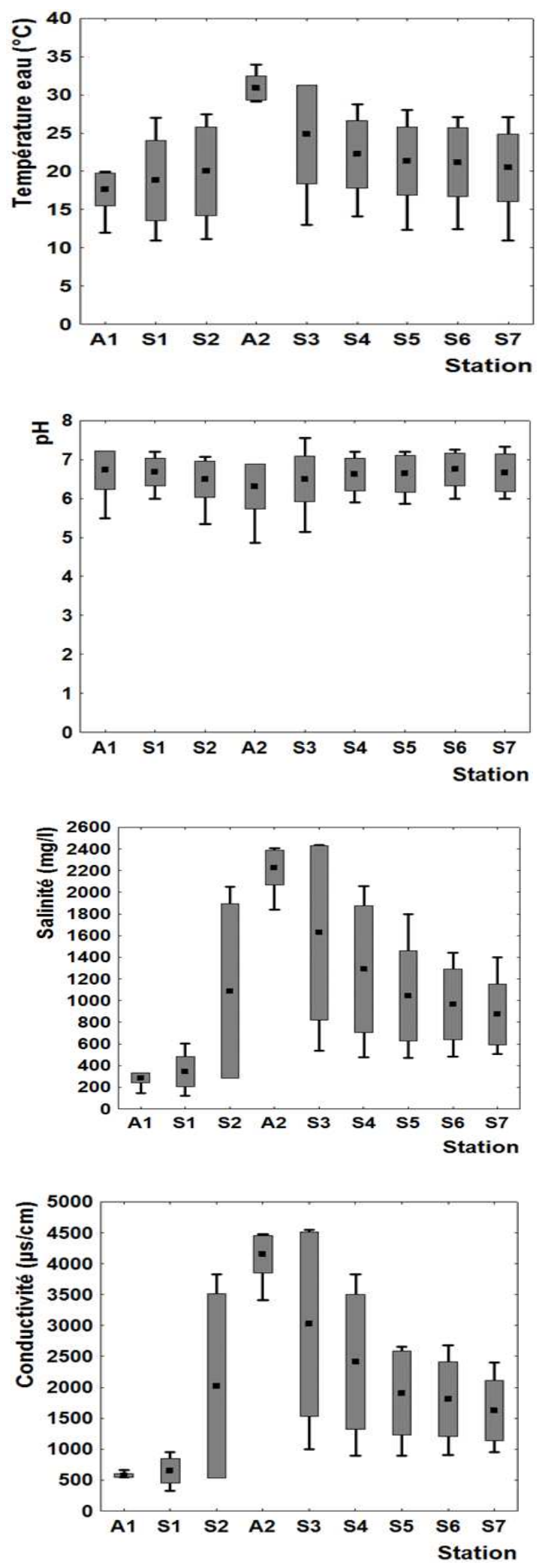

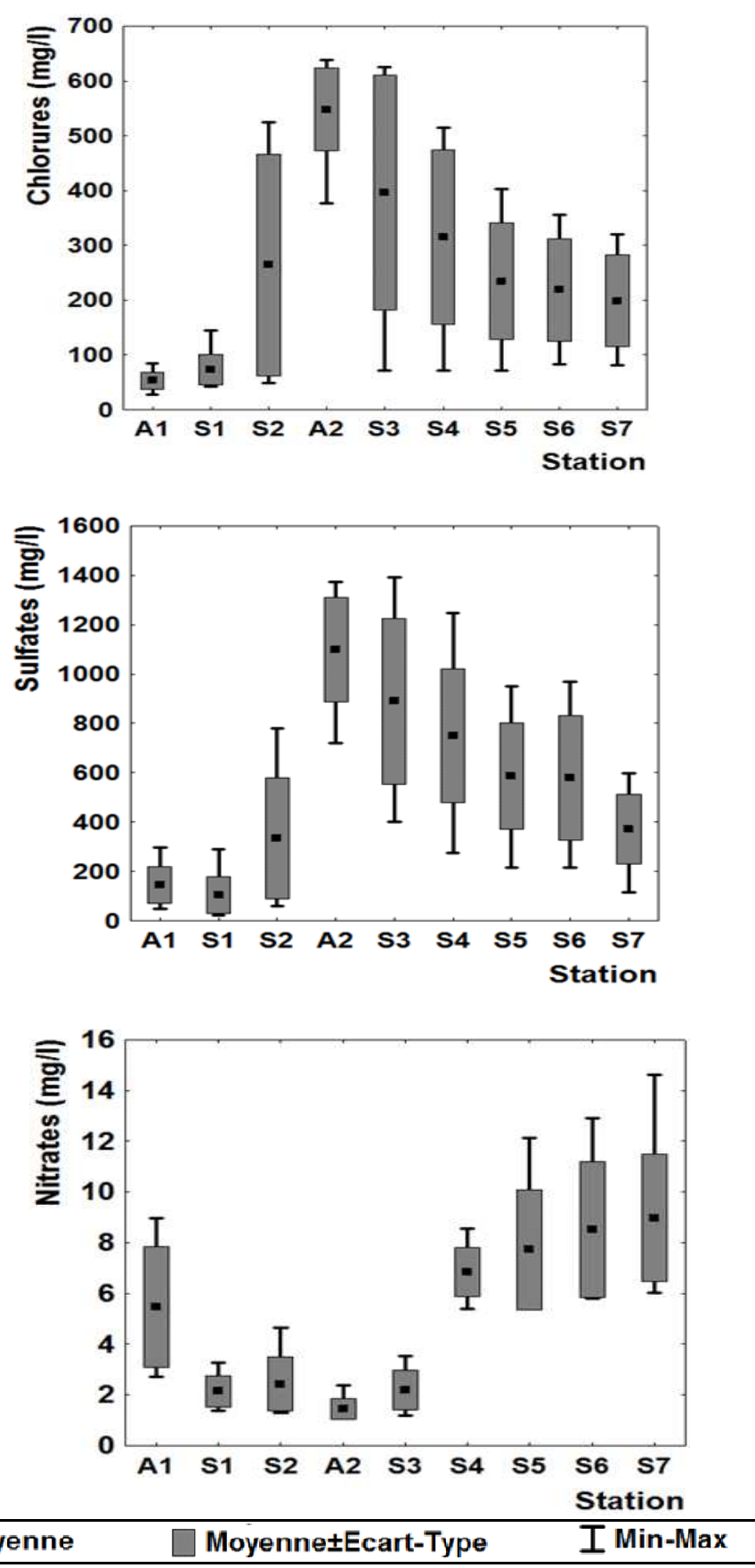

Figure 2: variations spatiales des concentrations des paramètres physico-chimiques. 
A. BEN MOUSSA et al. / Int. J. Biol. Chem. Sci. 6(6): 7096-7111, 2012
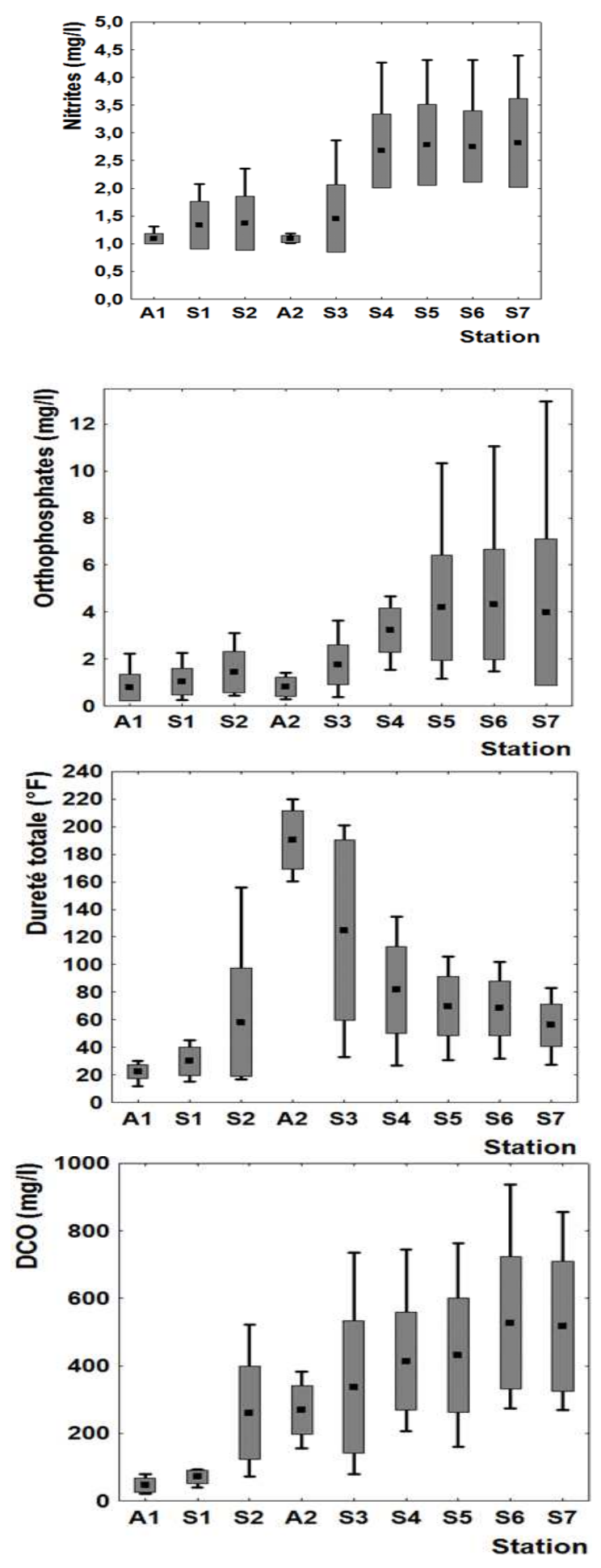
A. BEN MOUSSA et al. / Int. J. Biol. Chem. Sci. 6(6): 7096-7111, 2012
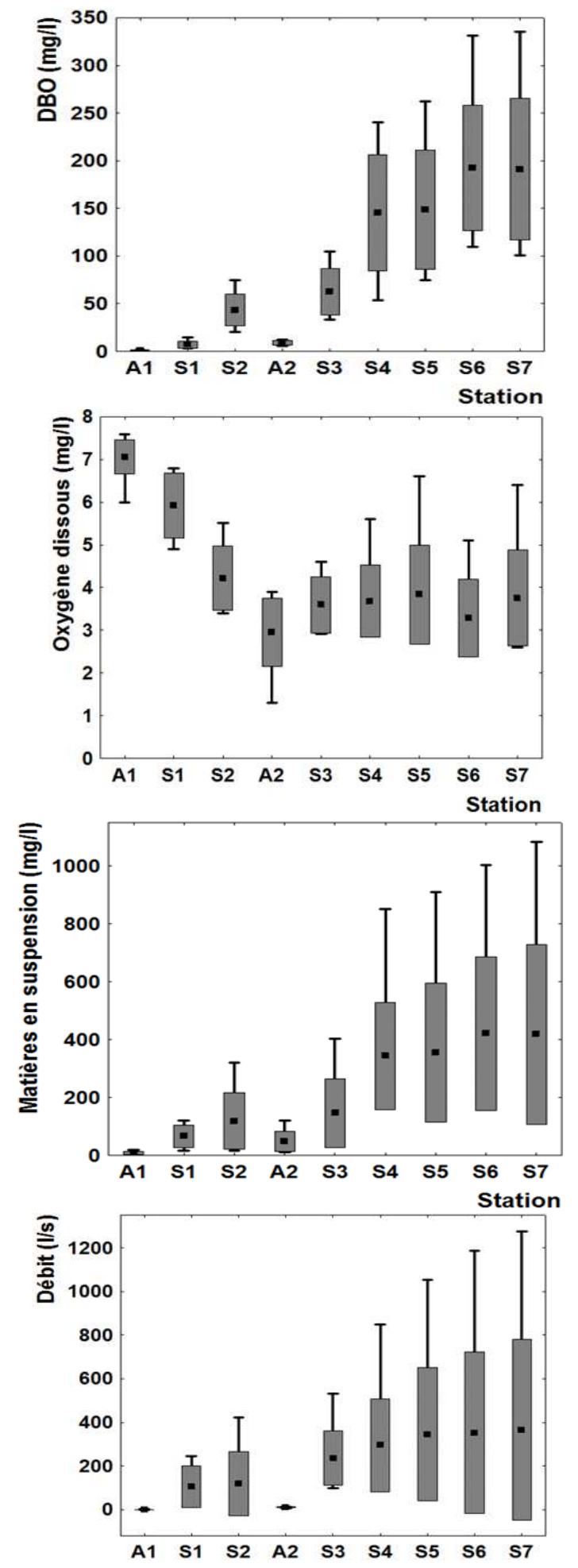

- Moyenne

$\square$ MoyennetEcart-Type

Station

Figure 3: Variations spatiales des concentrations des paramètres physico-chimiques (Suite). 
DCO : Demande Chimique en Oxygène; DBO : Demande Biologique en Oxygène.
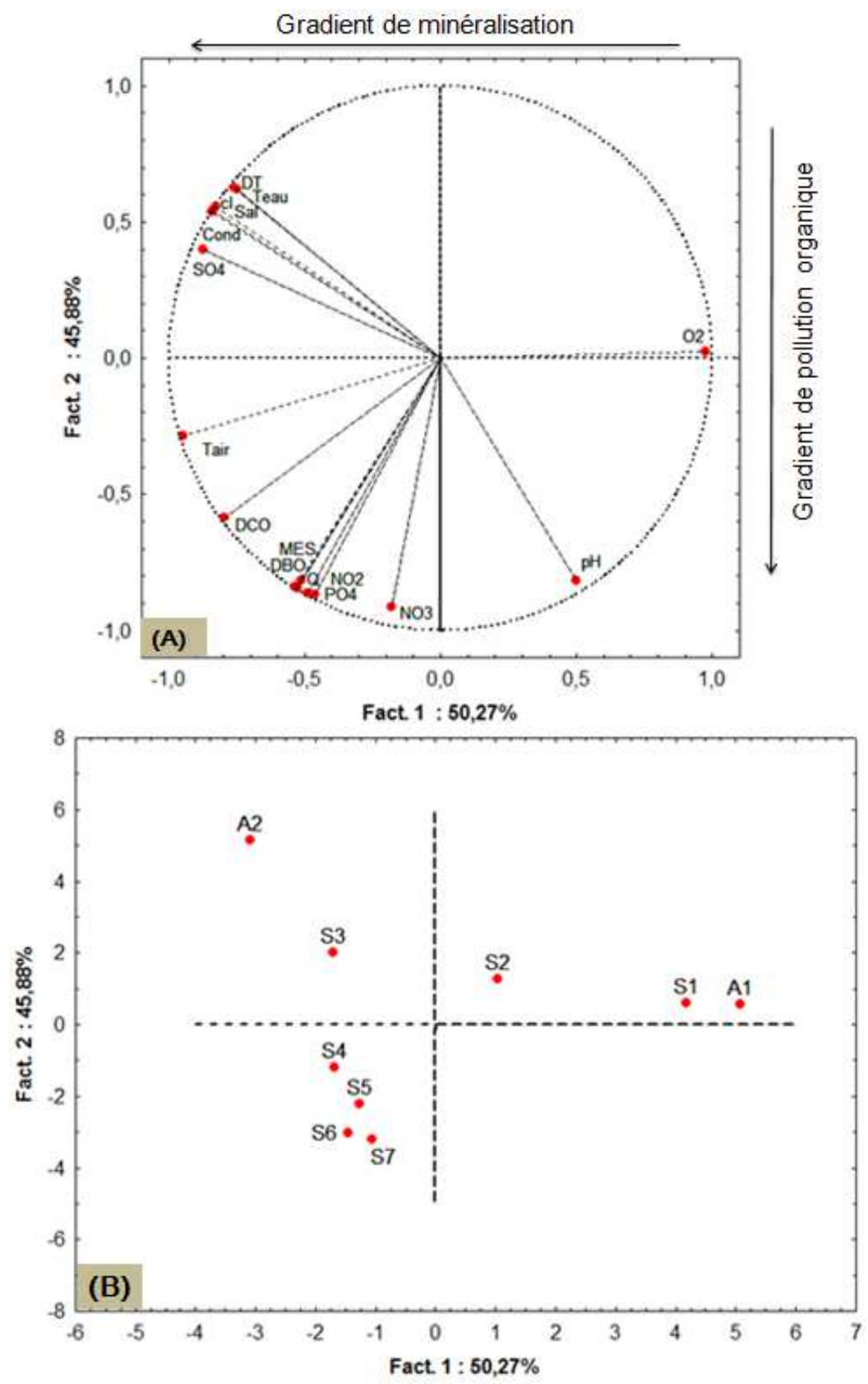

A: carte des paramètres écologiques (B): carte des stations; Température de l'air (T air); Température de l'eau (T eau); Potentiel Hydrogène (pH); Salinité (Sal); Conductivité (Cond); Chlorures (cl); Sulfates (SO4); Nitrates (NO3); Nitrites (NO2); Ortho phosphates (PO4); Dureté totale (DT); Demande Chimique en Oxygène (DCO); Demande Biologique en Oxygène (DBO); Oxygène dissous (O2); Matières en suspension (MES); Débit (Q).

Figure 4: Analyse en Composantes Principales des paramètres physico-chimiques. 


\section{DISCUSSION}

Le déversement des eaux thermales (source A2) réchauffe les eaux de l'Oued et explique les valeurs maximales enregistrées au niveau de S3. Cette augmentation brusque de la température de l'eau pourrait avoir un impact sur la qualité des eaux et donc sur la vie aquatique en aval. Le déversement, en aval de S3, des eaux de quelques sources naturelles (source Bousaid, Ain Rjal, Ain Oulili, ....) dilue les eaux de l'Oued et pourrait expliquer la diminution de la température (par rapport à S3) des eaux en aval. Les températures de l'eau mesurées sont inférieures à $35{ }^{\circ} \mathrm{C}$, considérée comme valeur limite indicative pour les eaux destinées à l'irrigation (Norme Marocaine, 2002a).

Le déversement direct des eaux de la source thermale dans l'Oued augmente la conductivité de ses eaux. Les résultats obtenus nous permettent de conclure que les eaux de l'Oued sont fortement minéralisées. Cette minéralisation dépasse largement celle montrée dans les Oueds Tizguit (Touabay et al., 2002), Boufekrane (Lamrani et al., 2011) et Ouislane (Aboulkacem, 2007). Comme ce paramètre est déterminé par la teneur en substances dissoutes, la charge ionique, la capacité d'ionisation, la mobilité et la température de l'eau (Derwich, 2010), les variations saisonnières observées pourraient s'expliquer aussi par le phénomène de dilution que connaît l'Oued suite aux précipitations en période hivernale (Nassali et al., 2005), contrairement à la période sèche (été et automne), où a lieu une élévation de ce paramètre accentuée par la forte évaporation (El Morhit et al., 2008) et favorisée par un bas débit de l'eau en faveur de la prédominance des eaux usées.

L'augmentation de la dureté totale des eaux le long de l'Oued vers l'aval pourrait s'expliquer, d'une part par le déversement des eaux thermales de la source Ain Hamma très dures $\left(190,58 \pm 21,04{ }^{\circ} \mathrm{F}\right)$ et d'autre part, par les apports anthropiques et la nature des roches traversées par l'eau (Aboulkacem, 2007). A titre de comparaison, la dureté totale au niveau de l'Oued Boufekrane et l'Oued Ouislane variait respectivement entre $26,4^{\circ} \mathrm{F}$ et $76^{\circ} \mathrm{F}$ et entre $30{ }^{\circ} \mathrm{F}$ et $116^{\circ} \mathrm{F}$, alors que dans l'Oued Bouishak elle est remarquablement élevée (supérieur à $200^{\circ} \mathrm{F}$ en moyenne) (karrouch, 2010). Le long de l'Oued, la dureté totale présente des valeurs, échelonnées sur la dernière classe (classe 7) de Nisbet et Verneaux (1970), qui correspond à des eaux très dures. Ces valeurs, relativement élevées et supérieures à $50^{\circ} \mathrm{F}$, indiquent une pollution chimique du milieu aquatique (karrouch, 2010).

L'évolution spatio-temporelle des chlorures dans les eaux de l'Oued présente la même allure que celle de la salinité, Ceci nous semble encore normal car l'ion chlorure est un paramètre qui nous renseigne sur la salinité du milieu. La nature géologique régionale, les lessivages des roches et des sols, les eaux domestiques et les eaux thermales de la source Ain Hamma (riche en chlorures) pourraient être à l'origine des ces fluctuations. Selon la classification proposée par Nisbet et Verneaux (1970), exceptes les eaux de la station S1 (échelonnées sur la classe 6), les eaux de l'Oued présentent des teneurs échelonnées sur la classe 7, qui correspond aux eaux plus ou moins polluées.

Les teneurs moyennes en sulfates dans les eaux de l'Oued dépassent fréquemment la norme fixée par la norme Marocaine relative à la qualité des eaux destinées à l'irrigation (Norme Marocaine, 2002a). D'après Nisbet et Verneaux (1970), les eaux de l'Oued en aval de S1 présentent des teneurs en sulfates échelonnées sur la dernière classe (classe 7 : $\left.\left[\mathrm{SO}_{4}{ }^{2-}\right]>250 \mathrm{mg} / \mathrm{l}\right)$, correspondant à des eaux très séléniteuse ou fortement polluées. En plus des eaux de la source thermale (très chargées) et de la nature géologique régionale; les résultats obtenus pourraient être liés au déversement des eaux usées domestiques (De Villers et al., 2005) et aux eaux des sources diluant les eaux en aval de S3. Les variations saisonnières des teneurs en sulfates pourraient s'expliquer en été par l'évaporation accentuée des eaux (Gouaidia, 2008) et par la 
prédominance des eaux de la source $\mathrm{A} 2$ et des eaux usées et, en hiver et en printemps, par le phénomène de dilution lié à l'augmentation du débit de l'Oued.

La contamination par les nitrates serait essentiellement liée aux lessivages des ions nitrates, abondamment répandus dans les sols suite à l'utilisation intense des engrais en agriculture et au fumier (Dumontier et al., 1995). Les rejets des eaux usées et les apports des eaux souterraines (sources d'eau) contaminées par l'infiltration de ces composés à partir des terres agricoles (Neal et al., 2000a) pourraient contribuer à cette contamination. L'augmentation des teneurs en hiver $(14,62 \mathrm{mg} / \mathrm{l}$ en décembre) est liée à l'accentuation du phénomène du lessivage des sols (Neal et al., 2000b) et au déversement, sans aucun traitement préalable, des margines dans l'Oued en décembre 2010 et janvier 2011. La diminution des teneurs en été (moins de 7,38 mg/l) et en automne (moins de 7,43 $\mathrm{mg} / \mathrm{l}$ ) pourrait s'expliquer par la diminution de la pollution diffuse (Neal et al., 2000b). Ces changements saisonnières sont en accord avec ceux enregistrés dans les cours d'eaux de Meknès (Aboulkacem, 2007; El addouli, 2010; Karrouch, 2010).

L'augmentation des teneurs en orthophosphates dans les stations en aval pourrait s'expliquer par l'impact des rejets domestiques chargés en orthophosphates. Les teneurs enregistrées restent inférieures à celles enregistrées par Karrouch (2010) au niveau des eaux des Oueds Boufekrane, Ouislane et Bouishak, et supérieures aux teneurs enregistrées dans les Oueds Merazig et Guenniche en Tunisie (Hammami et al., 2005). L'élévation de ces teneurs en hiver pourrait être liée à l'accentuation du lessivage des sols (Abba et al., 2010).

L'augmentation de l'amont vers l'aval de l'Oued de la Demande Chimique en Oxygène et de la Demande Biologique en Oxygène pourrait être liée au degré croissant de la pollution en aval. L'évolution saisonnière de ces parametres pourraient s'expliquer en hiver, par les rejets importants et inquiétant des margines en décembre 2010 et janvier 2011 (Foutlane et al., 2002) et par les lessivages des sols. En été et en automne, la diminution importante du débit de l'Oued associée à la prédominance des eaux usées pourraient être aussi à l'origine des teneurs enregistrées au cours de ces deux saisons.

Selon la Norme Marocaine relative à la qualité des eaux superficielles (Norme Marocaine, 2002b) et selon les teneures enregistrées en oxygène dissous; les eaux de $\mathrm{S} 1$, bien oxygénées durant la période d'étude, sont classées dans la grille de bonne qualité. Alors que, les eaux des autres stations sont de qualité moyenne à mauvaise en été et en automne, et de qualité moyenne en hiver et en printemps. Les résultats obtenus en été et en automne pourraient être attribués, essentiellement, à l'effet des températures élevées des eaux, à la prédominance des eaux usées et à la diminution importante du débit de l'Oued. Ceci pourrait être en faveur d'une multiplication bactérienne importante et donc de la consommation d'oxygène. Le même résultat (teneur faible en oxygène) a été enregistré dans les deux Oueds Guenniche et Hathat (en Tunisie) chargées en matière organiques (Hammami et al., 2005). L'augmentation des teneurs en oxygène dissous en hiver et en printemps pourrait être attribuée à un ensemble de facteurs, entre autres la diminution de la température de l'eau (Makhoukh et al., 2011) et l'augmentation du débit de l'Oued et donc à un brassage important de la masse d'eau. Les teneurs faibles enregistrées dans la source A2 sont liées à l'effet de la température élevée de ses eaux.

La comparaison des teneurs en matières en suspension (MES) obtenus avec la Norme Marocaine fixée à $1000 \mathrm{mg} / \mathrm{l}$, place les eaux de l'Oued dans la grille de bonne à moyenne (Norme Marocaine, 2002b). En hiver, les teneurs moyennes plus élevées en MES (surtout dans les quatre dernières stations) pourraient être liées au déversement inquiétant des margines dans ces stations et aux périodes des crues. Alors qu'en été et en 
automne, les valeurs enregistrées pourraient s'expliquer par un débit faible de l'Oued et par la prédominance des eaux usées.

Les résultats obtenus par l'ACP concordent avec les investigations effectuées sur le terrain. Les stations situées en amont sont loin de toute source de pollution, les stations en aval sont menacées par les rejets polluants : plus en se rapprochant de l'aval vers $\mathrm{S} 3$, les eaux deviennent de plus en plus minéralisées (Impact des rejets de la source thermale), et plus en s'éloignant de S3 vers l'aval de l'Oued, la pollution organique devient importante (Impact des domestiques de la ville).

\section{Conclusion}

A la lumière de ces résultats, nous enregistrons une dégradation inquiétante de la qualité de l'eau de l'Oued Khoumane, particulièrement dans sa partie soumise aux rejets des eaux thermales de la source Ain Hamma Moulay Idriss et aux rejets domestiques de la ville Moulay Idriss Zerhoun.

L'Analyse en Composantes Principales des données physico-chimiques nous a permis de faire ressortir la corrélation existante entre les différents paramètres et de différencier une typologie de la qualité de l'eau dans la région étudiée: zone plus oxygénée (plus en amont), zone avec un degré de pollution moyen (amont de la source thermale Ain Hamma Moulay Idriss), zone plus minéralisée (juste en aval de la confluence des eaux de l'Oued avec celles de la source thermale) et une quatrième zone, plus chargées en matière organique, s'étalant de la partie de l'Oued recevant les rejets domestiques de la ville moulay idriss Zerhoun vers l'aval.

Les résultats obtenus dans cette étude font ressortir une situation alarmante de la qualité des eaux de l'Oued; ce qui pourrait avoir des effets néfastes sur la faune et la flore de l'Oued, sur les sols irrigués par ces eaux et aussi sur la santé des populations environnantes.
Pour remédier à ce problème, nous recommandons un aménagement de la source thermale Ain Hamma Moulay Idriss, un réseau d'assainissement des eaux usées brutes adéquat et une gestion convenable des rejets émis par les huileries (activité économique principale de la population de Zerhoun).

\section{RÉFÉRENCES}

Abba E, Belghyti D, El Ibaoui H, Benabid M. 2010. Contribution to the physicochemical study of Oued Sidi Rachid river in the middle Atlas, Morocco. ScienceLib. Editions Mersenne, 2: 1-15.

Aboulkacem A. 2007. Étude hydrobiologique comparative des oueds Boufekrane et Ouislane à la traversée de la ville de Meknès. Impact sur la Sante et l'Environnement. Thèse d'Etat, Facultés des Sciences, Université Moulay Ismail, Meknès (Maroc), p. 159.

Aboulkacem A, Chahlaoui A, Soulaymani A, Rhazi-filali F, Benali D. 2007. Étude comparative de la qualité bactériologique des eaux des oueds Boufekrane et Ouislane à la traversée de la ville de Meknès (Maroc). Rev. Microbiol. Ind. San. Environn., 1(1): 1022.

Alibou J. 2002. Impacts des changements climatiques sur les ressources en eau et les zones humides du Maroc. Table Ronde Régionale en Méditerranée Athènes, Grèce, p1-39.

Bouaicha R, Benabdelfadel A. 2010. Variabilité et gestion des eaux de surface au Maroc. Sécheresse, 21(1): 1-5.

Bricha S, Ounine K, Oulkheir S, El haloui N, Attarassi B. 2007. Étude de la qualité physico-chimique et bactériologique de la nappe phréatique M'nasra (Maroc). Afrique Science, 3(3): 391- 404.

Chahlaoui A. 1996. Étude hydrobiologique de l'oued Boufekrane (Meknès), Impact sur l'environnement et la santé. Thèse d'État, Facultés des Sciences, 
Université Moulay Ismaïl, Meknès (Maroc), p. 234.

Chahlaoui A, Cugny P, Lek S, Zaid A, Ramdani M. 1997. Analyse quantitative de la qualité de l'eau de l'oued Boufekrane, Maroc. Bull. Soc. Hist. Nat., 133: 71-76.

Derwich E, Benaabidate L, Zian A, Sadki O, Belghity D. 2010. Caractérisation physico-chimique des eaux de la nappe alluviale du haut Sebou en aval de sa confluence avec oued Fès. Larhyss Journal, 8: 101-112.

De villers J, Squilbin M, Yourassowsky C. 2005. "L'eau à Bruxelles" .Institut Bruxellois pour la Gestion de l'Environnement/Observatoire des Données de l'Environnement. Fiche 2: 116.

Dumontier M, Dethier M, Revaclier R, Balikungeri A. $1995 . \quad$ Étude hydrobiologique du bassin de l'Hermance. Bull. Mens. Soc. Linn. Lyon, 9: 411-422.

El addouli J. 2010. Qualité physico-chimique et biologique des eaux polluées réutilisées en agriculture dans la zone périculture de Meknès (Maroc). Thèse d'état, Facultés des Sciences, Université Moulay Ismaïl, Meknès (Maroc), p. 168.

El addouli J, Chahlaoui A, Berrahou A, Chafi

A, Ennabili A. 2009a. Qualité physicochimique et biologique de l'oued Ouislane au sein de la ville de Meknès (centre-nord duMaroc). Revue de l'Association Forum du Nord du Maroc, 4-5: 46-58.

El addouli J, Chahlaoui A, Berrahou A, Chafi A, Ennabili A, Karrouch L. 2009b. Influence des eaux usées, utilisées en irrigation, sur la qualité des eaux de l'oued Bouishak- région de Meknès (Centre-Sud du Maroc). Rev. Microbiol. Ind. San. Environn., 3(1): 56-75.

El addouli J, Chahlaoui A, Lamrani $\mathrm{H}$, Chafi A, Ennabili A. 2012. Aspect sanitaire et socioéconomique liés à la réutilisation des eaux usées en agriculture Région de
Meknès. ScienceLib Editions Mersenne, 4: 17.

El morhit M, Fekhaoui M, Serghini A, El blidi S, El abidi A, Bennaakam R, Yahyaoui A, Jbilou M. 2008. Impact de l'aménagement hydraulique sur la qualité des eaux et des sédiments de l'estuaire du Loukkos (côte atlantique, Maroc). Bull. Inst. Sci. Rabat, 30: 39-47.

Foutlane A, Saadallah M, Echihabi L, Bourchich L. 2002. Pollution par les margines et production d'eau potable. Cas de l'Oued Sebou au Maroc. Eastern Mediterranean Health Journal, 8(1): 10.

Gouaidia L. 2008. Influence de la lithologie et des conditions climatiques sur la variation des parametres physico chimiques des eaux d'une nappe en zone semi aride, cas de la nappe de Meskiana nord-est Algérien. Thèse de Doctorat, Faculté des sciences de la Terre, Université Badji Mokhtar-Annaba (Algérie), p. 199.

Hammami J, Brahim M, Gueddari M. 2005. Essai d'évaluation de la qualité des eaux de ruissellement du bassin versant de la lagune de Bizerte. Bulletin de l'Institut National des Sciences et Technologies de la Mer Salammbô, 32: 69-77.

Karrouch L. 2010. Bio-évaluation de la qualité des eaux courantes de la région Meknès (Centre- Sud, Maroc) - Impact sur l'environnement et la sante. Thèse doctorat es sciences en biologie. Facultés des Sciences, Université Moulay Ismaïl, Meknès, Maroc, p. 216.

Karrouch L, Chahlaoui A. 2007. Évaluation de la qualité biologique des eaux de l'oued Boufekrane (Meknès, Maroc). $2^{\text {ème }}$ Colloque International sur l'Eau et l'Environnement : Sidi Fredj le 30 et 31 janvier, 224-235.

Karrouch L, Chahlaoui A. 2009. Bioevaluation of the quality of water of the Boufekrane River (Meknes, Morocco). Biomatec Echo, 3(6): 6 - 17.

Lamrani H, Chahlaoui A, El addouli J, Ennabili A. 2011. Évaluation de la 
qualité physicochimique et bactériologique de l'oued Boufekrane au voisinage des effluents de la ville de Meknès (Maroc). ScienceLib Éditions Mersenne, 3: 15.

Lamrani H, Chahlaoui A, El addouli J, Ennabili A. 2012. Etude de la qualité des eaux de l'oued Boufekrane et application de l'Analyse en Composantes Principales. ScienceLib Éditions Mersenne, 4: 16.

Makhoukh M, Sbaa M, Berrahou A, Van Clooster M. 2011. Contribution a l'étude physico-chimique des eaux superficielles de l'Oued Moulouya (Maroc oriental). Larhyss Journal, 9: 149-169.

Nassali H, Ben Bouih H, Srhiri A, Dhahbi M. 2005. Influence des rejets des eaux usées sur la composition des eaux de surface et des sédiments superficiels du lac Merja Fouarate au Maroc. Afrique Science, 1(1): 145 - 165 .

Neal C, Jarvie HP, Howarth SM, Whitehead PG, Williams RJ, Neal M, Harrow M, Wickham H. 2000a. The water quality of the River Kennet: initial observations on a lowland chalk stream impacted by sewage inputs and phosphorus remediation. The Science of the Total Environnement, 251-252: 477- 495.

Neal C, Neal M, Wickham H, Harrow M. 2000b. The water quality of a tributary of the Thames, the Pang, southern
England. The Science of the Total Environment, 251-252: 459-475.

Nisbet M, Verneaux J. 1970. Composantes chimiques des eaux courantes. Ann. Limnol., 6(2):161-190.

Norme Marocaine de qualité des eaux. 2002a. Arrêté conjoint du ministre de l'équipement et du ministre charge de l'aménagement du territoire, de l'urbanisme, de l'habitat et de l'environnement $\mathrm{n}^{\circ}$ 1676-01 du 10 chaabane 1423 (du 17 octobre 2002) portant fixation des normes de qualité des eaux destinées a l'irrigation. Bulletin Officiel Maroc, $\mathrm{N}^{\circ} 5062$.

Norme Marocaine de qualité des eaux. 2002b. Arrêté conjoint du Ministre de l'équipement et du Ministre chargé de l'aménagement du territoire, de l'urbanisme, de l'habitat et de l'environnement $\mathrm{n}^{\circ} 1275-01$ du 10 Chaabane 1423 (17-10-2002) définissant la grille de qualité des eaux de surface. Bulletin Officiel Maroc, №5062.

Rodier J. 2009. L'Analyse de l'Eau (9édn). Dunod: Paris, France.

Touabay M, Aouad N, Mathieu J. 2002. Étude hydrobiologique d'un cours d'eau du Moyen-Atlas : l'oued Tizguit (Maroc). Ann. Limnol., 38(1): 65-80. 